\title{
Vacuum-Flow Steel Degassing
}

\author{
A Journal of Metals Exclusive from Germany \\ by F. Weston Starratt
}

\begin{abstract}
Still in the development stage by one of the Ruhr's oldest steel plants, this new vacuum-flow steel degassing technique appears to hold great promise for low-cost degassing of steel for large forgings and castings.
\end{abstract}

$T$ HE Henrichshütte, one of the oldest steel plants in Germany's Ruhr was founded more than a century ago by Count Henrich zu Stolberg-Wernigerode. Passing through various ownerships, the plant became a part of Ruhr AG during the late twenties. Severely damaged during World War II, the plant later endured the dismantling of a large open-hearth furnace, the electric furnaces, and a plate mill. The steel plant and the forging and casting shops have been completely modernized in recent years, and today the forging shop is one of the largest and most modern in Germany.

The plant's present production program is concentrated in large forgings, with castings up to 200 tons and medium plates also occupying an important position. Forgings made from ingots weighing up to 180 metric tons are in the form of crank shafts, turbine shafts, rotors, back-up rolls for steel rolling mills, wheels, axels, and many other products.

With such a program of heavy forgings, the problem of flaking, resulting from contamination by hydrogen and other gases, has been of primary concern to the firm and resulted in a research program largely oriented toward vacuum degassing. Work was carried foreward in connection with the Heraeus firm of Hanau, Germany, the builder of vacuum pumps and various metallurgical equipment.

\section{Operation appears effective and low in cost}

The vacuum-flow steel degassing developed at Heinrichshütte has now been in commercial operation for over 6 months with apparant success, although operating figures have not yet been released by the firm.

Capital costs for the installation are obviously quite low, since all that is required is the small vacuum container with connecting pipes and the vacuum pumps with auxiliary equipment.

The apparatus is reportedly capable of bringing the hydrogen, nitrogen, and oxygen content of all types of steel to as low levels as are obtained in vacuum casting techniques. The operation is carried out in normal steel ladles of capacities between 60 and 80 metric tons.

\section{For all types of steel}

This vacuum degassing process is adaptable to all types of carbon steel-rimming and killed-as well as most alloy steels. At the Heinrichshütte, it is used both on open-hearth and electric-furnace steels, but it can be adapted to other steel-making practice. In all cases a special effort is made to remove furnace slag, because of its high oxygen content, but with some alloy steels a special slag is made to cover the steel in the ladle during the degassing operation, thereby preventing excessive oxidation.

The degassing operation in the 60 to 80 -ton ladles requires from 12 to $15 \mathrm{~min}$. The cylinderical container with two pipes protruding is lowered into the steel bath in the ladle until the pipes extend below the level of the metal. The apparatus is shown in Fig. 1, which is a rough drawing by the author.

The vacuum created in the chamber causes the metal level to rise in both pipe, but small quantities of argon gas are blown into the lower end of one of the protruding pipes, thereby causing a flow of metal up one of the connecting pipes, through the container, and down the other pipe. A vacuum of 0.1 $\mathrm{mm} \mathrm{Hg}$ is maintained in the container. Between the container and the vacuum pumps is a cyclone dust catcher, as shown in Fig. 1.

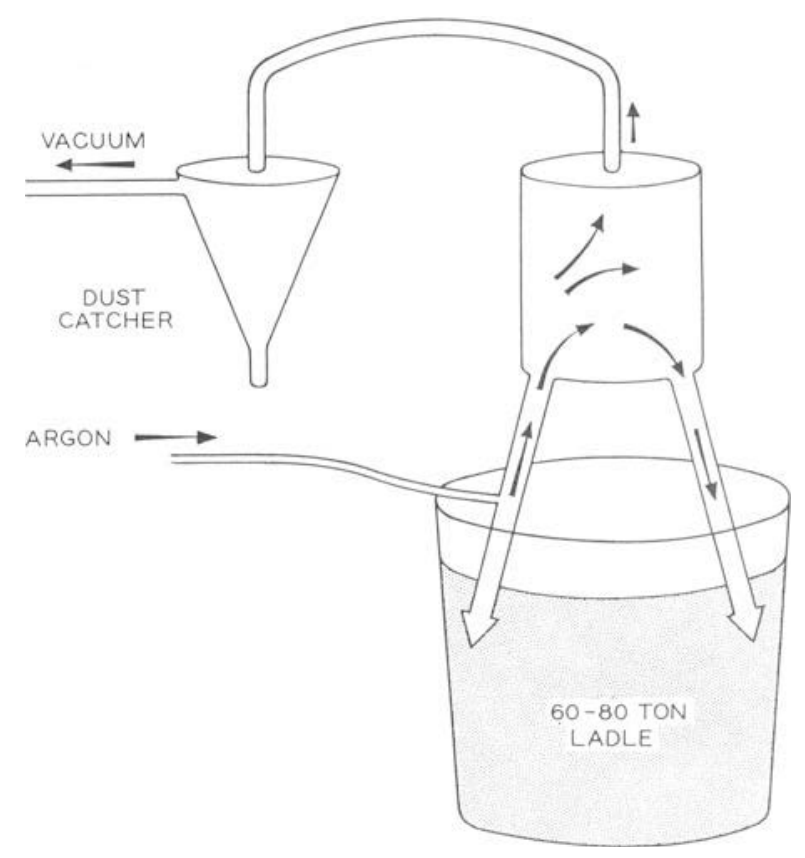

Schematic diagram of the vacuum-flow steel degassing apparatus.

The vacuum pumps consist of an initial series of three Roots pumps in parallel with a pumping capacity of $28,000 \mathrm{cu} \mathrm{m}$ per hr. This is followed by a second series of two Roots pumps in parallel with a capacity of $6000 \mathrm{cu} \mathrm{m}$ per $\mathrm{hr}$, and one further Roots pump of $1000 \mathrm{cu} \mathrm{m}$ per hr capacity. The final pump is a water-ring pump, with an eccentric paddlewheel-type rotor; it has a capacity of $750 \mathrm{cu}$ m per $\mathrm{hr}$ and exhausts gases through a pipe into the atmosphere.

The degassing process may be continued until the proper gas level has been reached. Continuous samples of gas are taken from the final pump and analyzed in an Orsat apparatus. It is on the basis of this analysis that the end point of the vacuum degassing process is judged.

While little information has been released on this new process for the removal of gases from steel, it is certainly worth noting, for it appears to be an effective, low-cost technique for producing flakefree large forgings and castings. 